NOTE ON LMC X-4

\author{
TIBOR J. HERCZEG \\ Department of Physics and Astronomy \\ University of Oklahoma \\ Norman, Oklahoma 73019, U.S.A.
}

\begin{abstract}
Orbital and stellar parameters of the massive X-ray binary LMC X-4 are rediscussed with particular attention to a possible "higher mass" $\left(>1.4 \mathrm{M}_{\odot}\right)$ neutron star in the system.
\end{abstract}

\title{
Introduction: Neutron Star Masses
}

The early impression that neutron stars have a "standard" mass at $1.4 \mathrm{M}_{\odot}$ has been somewhat qualified by recent mass determinations: $1.4 \mathrm{M}_{\odot}$ is still a good gauge but the best masses, obtained by high accuracy pulse timing and relativistic solutions, seem to "cluster" in the range 1.30 to $1.45 \mathrm{M}_{\odot}$.

$$
\begin{array}{ll}
\text { PSR } 1913+16 & 1.44+1.39\left(\mathrm{M}_{\odot}\right), \text { Taylor \& Weisberg, error }<0.01 \mathrm{M}_{\odot} \\
1855+09 & 1.27+\text { WD, Ryba \& Taylor, error }+0.27 /-0.16 \mathrm{M}_{\odot} \\
\text { PSR } 1534+12 & 1.36+1.32\left(\mathrm{M}_{\odot}\right), \text { Wolszczan, error } \pm 0.03 \mathrm{M}_{\odot}
\end{array}
$$

In the cases of PSR $2303+46$ and $2127+11 \mathrm{C}$ we only have the sum of the masses, $2.9 \pm 0.03 \mathrm{M}_{\odot}$ resp. $2.7 \mathrm{M}_{\odot}$, from the relativistic apsidal rotation (Lyne \& Bailes, resp. Prince et al.). We did not list the controversial system PSR 0021-72A; here the masses may also be close to $1.4 \mathrm{M}_{\odot}$. As to the X-ray binaries, they leave us with much larger error limits; the best solutions for Her X-1 suggests a neutron star mass between 1.3 and $1.4 \mathrm{M}_{\odot}$. (References at the end of the paper.)

The question arises, do some of the neutron stars in massive X-ray systems have masses higher than $1.4 \mathrm{M}_{\odot}$ ? After all, in an accretion-powered system the compact object may well gain a few $0.1 \mathrm{M}_{\odot}$. Where are these systems? Joss and Rappaport (1983) listed 3 massive systems with possibly "overmassive" neutron star components: 4U 0900-40, 4U 1538-52 and LMC X-4, a well studied system where, to quote Joss and Rappaport, all the required ingredients for an orbital analysis are now available -including, we may add, a reasonably well known distance $( \pm 10 \%)$. Their review gives the most probable neutron star mass for LMC X-4 as $1.7 \mathrm{M}_{\odot}$ although with the 
considerable uncertainty of $+1.9 /-1.0\left(\mathrm{M}_{\odot}\right)$.

\section{Observational Background}

LMC X-4 has been optically identified by Chevalier and Ilovaisky. The noncompact component is an 07 star of about -4.5 abs. vis. magnitude; the period is 1.408 days. The main spectroscopic study is -- as in the case of so many X-ray binaries -- by Hutchings, Crampton and Cowley (1978). Their elements are the basis for the entries in the "Eighth Catalog" by Batten et al., they give $\mathrm{K}_{1}=60 \mathrm{~km} / \mathrm{s}$, derived from the He-lines. Of particular interest was the HeII emission line at $\lambda 4686$ which apparently moved in phase with the expected X-ray source and had a large radial velocity amplitude, around 475 $\mathrm{km} / \mathrm{s}$; a good estimate of the mass ratio can be derived. Further observations include (besides spectra by Chevalier and Ilovaisky used in the solution mentioned above) observations by Hiltner and Pedro and another, yet unpublished, set of some 30 spectra of more modest accuracy taken 1986-87 with the ESO $1.5 \mathrm{~m}$ telescope by Herczeg. These spectra were taken with the intention to follow up the possibility that the $K_{1}$ amplitude may be less than $60 \mathrm{~km} / \mathrm{s}$, possibly closer to $50 \mathrm{~km} / \mathrm{s}$. Since a change alone in $\mathrm{K}_{1}$ of $2 \mathrm{~km} / \mathrm{s}$ would imply a change in the neutron star mass of about $0.08 \mathrm{M}_{\odot}$, this value is rather critical.

Kelley et al. discovered X-ray pulses of $13.5 \mathrm{sec}$. length and obtained a (circular) orbit of $(30 \pm 5)$ lt-s projected radius; an improved X-ray orbit was derived by Pietsch et al. with $(26.0+0.5) 1 t-s$ projected radius. The resulting new radial velocity amplitude may now supersede the ingenious attempt to use the HeII emission for obtaining the mass ratio.

The LMC X-4 system shows X-ray eclipses albeit with a not very well defined eclipse half-angle around 16-20 deg. It can be used to a rather crude estimate of the inclination ranging somewhere between $58^{\circ}$ and $90^{\circ}$; yet a sharper estimate, $70^{\circ}$ to $80^{\circ}$, can probably be maintained.

Spectra reaching beyond the blue-green range, as do the ESO spectra, show very strong [OIII] lines at $\lambda 5007$ and $\lambda 4959$, almost certainly circumstellar lines. They may indicate considerable mass loss from the system, possibly rendering the early type component undermassive. (Catalogue values for the mass of a normal 07 star are 20 to $28 \mathrm{M}_{\odot}$.) A particularly strong mass loss may be connected with the O-star nearly filling its Roche surface -- even an overflow of the Roche lobe is not entirely out of the question as a main source of accretion.

\section{Solutions}

Earlier estimates for the neutron star mass were based on the good agreement between the radial velocities of the HII emission and the first X-ray orbit; they give $K_{x}=475$ $\mathrm{km} / \mathrm{s}$ resp. $465 \mathrm{~km} / \mathrm{s}$. Relying more on the EXOSAT observations changes the picture somewhat. 
Instead of a formal solution with those very large error bars we present a "chart" representing various partial solutions as functions of two parameters. This may give some insight into the structure of the solutions and enable us to make at least some useful, qualitative statements, such as "the mass of the compact component has to be above $1.4 \mathrm{M}_{\odot}$."

Assuming that the solution depends more on the optical than the X-ray amplitude and taking $K_{x}=410 \mathrm{~km} / \mathrm{s}$ as a weighted mean of the data, we obtain the following skeleton chart of the solutions. (Masses in $\mathbf{M}_{\odot}$, in parenthesis the 07 star values.)

\begin{tabular}{l|c|c|}
\multicolumn{1}{c|}{$\begin{array}{c}\mathrm{i}=90^{\circ} \\
(\min . \text { mass })\end{array}$} & $\begin{array}{c}\mathrm{i}=60^{\circ} \\
(\max . \text { mass) }\end{array}$ \\
\hline $\mathrm{K}_{1}=60 \mathrm{~km} / \mathrm{s}$ & $\begin{array}{c}\mathrm{M}=1.93 \\
(13.2)\end{array}$ & $\begin{array}{c}\mathrm{M}=2.98 \\
(20.3)\end{array}$ \\
\hline $\mathrm{K}_{1}=50 \mathrm{~km} / \mathrm{s}$ & $1.54(12.7)$ & $2.38(19.5)$ \\
\hline $\mathrm{K}_{1}=40 \mathrm{~km} / \mathrm{s}$ & $1.18(12.1)$ & $1.82(18.7)$ \\
\hline
\end{tabular}

The mass of the early type component is a weak gauge of the solution. Nevertheless, masses around $12-13 \mathrm{M}_{\odot}$ for the 07 star are very difficult to accommodate considering any plausible physical description of the system.

We are following a "limited plan" of the mass determination: selecting plausible values of the mass-function, say corresponding to $\mathrm{K}_{1}=55 \mathrm{~km} / \mathrm{s}$, and then varying two parameters, for instance, the O-star mass and the inclination or the mass ratio and inclination, we can easily convince ourselves that the probability of $M_{x}>1.4$ turns out to be a near certainty, and even $M_{x}>1.5$ leads to very high probabilities, above 0.8 .

\section{Conclusion}

It is obvious that we cannot describe an X-ray binary with an accuracy similar to the best determined cases of binary radio pulsars. The comparatively well studied system LMC X-4 enables us to marshal evidence in favor of the neutron star component being somewhat (a few $0.1 \mathrm{M}_{\odot}$ ?) above the value of $1.4 \mathrm{M}_{\odot}$. It is certainly falling far short of black hole masses, in spite of the earliest estimates yielding an intriguing 2-4 $\mathbf{M}_{\odot}$.

ACKNOWLEDGEMENTS. I wish to thank Virginia Trimble for procuring important references in the literature and Loretta McKibben for her essential help in preparing the manuscript. 


\section{REFERENCES}

Overview: S. Rappaport, P. C. Joss in "Accretion Driven Stellar X-ray Sources" (eds. W. H. G. Lewin, E. P. J. Van den Heuvel), Cambridge U. Press 1983 Spectroscopy: C. Chevalier, S. A. Ilovaisky, A Ap 59, L9, 1977; J. Hutchings, D. Crampton, A. Cowley, Ap J 225, 548, 1978.

Eclipses: F. Li, S. Rappaport, A. Epstein, Nature 271, 37, 1978; N. E. White, Nature 271, 38, 1978.

X-ray pulses and orbit: R. L. Kelley et al. Ap J 264, 568, 1983; W. Pietch et al., Space Sci. Rev. 40, 371, 1985, see also M. Pakull et al, Space Sci. Rev. 40, 379, 1985.

Pulsar masses: A. Wolszczan, Nature 350, 688, 1991 (1534 + 12); M. F. Ryba, J. H. Taylor, Ap J 371, 739, 1991 (1855 + 09); J. H. Taylor, J. M. Weisberg, Ap J 345, 434, 1989 (1913 + 16); T. A. Prince et al, Ap J 374, L41, 1991 $(2227+11$ C); A. G. Lyne, M. Bailes, $M N, 246$, P15, $1990(2303+46)$. 